\title{
Adaptive generalized space shift keying
}

\author{
Konstantinos Ntontin ${ }^{1 *}$, Marco Di Renzo ${ }^{2}$, Ana Perez-Neira ${ }^{1}$ and Christos Verikoukis ${ }^{1}$
}

\begin{abstract}
In this article, we propose a closed-loop precoding method for the Generalized Space Shift Keying (GSSK) modulation scheme, suitable for Multiple-Input-Single-Output (MISO) systems and denoted as adaptive GSSK (AGSSK), which achieves transmit-diversity gains in contrast to GSSK. For the case of a perfect feedback channel, we analytically show that for three and four antennas at the transmitter and rates 1 and 2 bits per channel use (bpcu), respectively, a full transmit-diversity can be achieved without reducing the achievable rate. For higher number of transmit antennas and rates, the performance of the proposed scheme degrades due to the smaller average minimum Euclidean distance as the rate increases. Due to this, we, furthermore, propose an enhancing method for AGSSK which relies on the use of time-orthogonal shaping filters for the different constellation points. For the enhanced method, named as AGSSK with time-orthogonal signal design (AGSSK-TOSD), we analytically prove that it offers transmit-diversity gains which are greater than the number of active transmit antennas for any number of transmit antennas and supported rate. This is attained without any antenna subset selection technique, which alleviates the processing burden on the terminal side. Monte Carlo simulations show that AGSSK significantly outperforms GSSK in terms of average bit error probability (ABEP) and, moreover, for medium to high rates and practical signal-to-noise ratio (SNR) regions AGSSK-TOSD outperforms well-known feedback-based multiple-antenna schemes. This advantage of AGSSK-TOSD is further substantiated with an energy effficiency comparison over the conventional schemes for a target (uncoded) ABEP.
\end{abstract}

\section{Introduction}

\subsection{General background}

Spatial Modulation (SM) [1-3] is a recently introduced innovative single-RF Multiple-Input-Multiple-Method (MIMO) method, in which information is conveyed in two ways: (i) through a conventional signal constellation diagram, such as Phase Shift Keying (PSK) or Quadrature Amplitude Modulation (QAM), and (ii) through the use of a spatial constellation diagram ([3], Fig. one), thus offering a rate gain which scales with the binary logarithm of the number of transmit antennas. Depending on the number of transmit and receive antennas, it has been shown that SM can outperform well-known conventional multiple antenna technologies, such as the Vertical Bell Labs layered space-time architecture (VBLAST) [4] and Single-Input-Multiple-Output (SIMO) systems [2,5]. In generalized SM (GSM), the single RF restriction is relaxed by allowing more RF chains and, hence, more than one active antennas to be included in the design [6]. GSM

\footnotetext{
*Correspondence: constantinos.ntontin@cttc.es

1 Telecommunications Technological Centre of Catalonia (CTTC), Castelldefels, Spain

Full list of author information is available at the end of the article
}

can achieve the same rates as SM with a smaller number of transmit antennas, but at a cost of a lower average bit error probability (ABEP).

On the other hand, Space Shift Keying (SSK) is a simpler implementation of SM in which only the antenna indices are used as a mean to convey information, thus avoiding the use of signal constellation diagrams [7]. Its simplicity comes at a cost of requiring a higher number of transmit antennas than SM to achieve the same rates. In the same way as with GSM, more than one RF chains and, consequently, more than one active antennas can be used in the so-called generalized SSK (GSSK) scheme [8]. A peculiar feature of the SSK/GSSK modulation methods is that their diversity gains do not depend on the fading severity of the channels, as it is proven in [9] for the case of Nakagami- $m$ fading channels. This is in contrast to conventional systems that use PSK/QAM modulation to convey information.

\subsection{Enhancing the performance of conventional SM/SSK systems through transmit-diversity}

A drawback of the conventional open-loop SM/SSK schemes $[2,7]$ is that they can offer only receive-diversity gains, which is proven in [10] by an eigenvalue analysis.

\section{望 Springer}

(c) 2013 Ntontin et al: licensee Springer. This is an Open Access article distributed under the terms of the Creative Commons Attribution License (http://creativecommons.org/licenses/by/2.0), which permits unrestricted use, distribution, and reproduction in any medium, provided the original work is properly cited. 
Due to this, there have been several works in literature that improve the performance in the high-SNR region of those schemes through means of transmit-diversity, which can be achieved either by open or closed-loop design [11-23].

\subsubsection{Transmit-diversity for SM systems}

Regarding open-loop schemes that enable transmitdiversity gains for SM systems, the well-known spacetime block coding technology [24] is used in conjunction with SM in $[11,12]$, which results in transmit-diversity gains at a cost of requiring at least two active antennas and, consequently, two RF chains in contrast to the single-RF conventional SM. Sugiura et al. [13], introduced the Space-Time Shift Keying (STSK) modulation scheme, where SM is included as a special case, which encodes additional information bits onto dispersion matrices and exploits space-time coding. It provides a transmit-diversity gain that scales with the minimum between the number of transmit antennas and the number of time slots. A drawback is the linear decrease of the rate with the number of time-slots that are used in the encoding process. To solve this, the generalized version of STSK is proposed in [14] in which more than one active dispersion matrices are used at each time slot. This way, the rate is increased with respect to STSK at a cost of a higher encoding and decoding complexity. Regarding transmit-diversity achieving open-loop SM schemes that retain the important feature of requiring a singleRF chain, to the best of the authors' knowledge there is only the work in [15] in which the authors exploit the Complex Interleaved Orthogonal Design (CIOD) [25] to propose a SM-based scheme that achieves a transmit-diversity gain equal to two without sacrificing rate.

As far as closed-loop SM schemes are concerned, Yang et al. [16] proposes an adaptive single-RF SM scheme in which the receiver chooses at each time slot the most suitable modulation order for each of the possible transmit antennas based on the channel estimates and feeds the selected transmit mode to the transmitter. The same authors generalize their work in [17] where the possibility of selecting the best subset of antennas to implement SM is included in the design apart from the modulation order. A simpler version of the idea presented in [17], where only the subset of antennas is selected and not the possible transmit mode from each of the antennas of the selected subset, was proposed in [18]. Finally, Ma et al. [19] introduced the so-called adaptive joint mapping generalized SM (AJM-GSM) in which the incoming data bits are jointly mapped to both the signal and spatial symbols in a dynamic way at each time slot, based on the channel state information (CSI) at the receiver.

\subsubsection{Transmit-diversity for SSK systems}

Transmit-diversity schemes for SSK systems is an area with limited contributions. In particular, for open-loop systems the authors in $[20,21]$ proposed the use of time-orthogonal shaping filters for each of the transmit antennas. This way, the achievable transmit-diversity gain with the proposed design, which is denoted as SSK-time orthogonal signal design (SSK-TOSD), is equal to two. The realization of practical time-orthogonal shaping filters is discussed in [26]. In addition, the same authors apply in [22] their time-orthogonal shaping filter design to GSSK systems and they prove that the achievable transmit-diversity gains are equal to the minimum number of different channel links among all the possible pairs of constellation points. Hence, there is a significant loss of transmit-diversity and consequently of performance if the constellation points include many common channel links.

As far as closed-loop schemes are concerned, to the best of the authors' knowledge there is only the work of [23] in which the authors introduced a feedback-based method suitable for $2 \times 1$ SSK systems where a phase variable is fed to the transmitter. For perfect feedback, it is proven that the transmit-diversity gain achieved is equal to 2 .

\subsection{Contribution}

Motivated by the small amount of previous work related to feedback-based SSK/GSSK systems that offer transmitdiversity gains, we propose a feedback-based precoding technique, which is shown to enhance significantly the performance of these systems, both in terms of diversity and coding gain. More specifically, our work is focused on the GSSK modulation for MISO systems and we assume that the phases of the channel links can be made available to the transmitter by considering a low-delay feedback link. The reason for studying MISO systems is that they have a high practical importance due to widespread use of one-antenna pocket-sized terminals. In these systems, an effective way to improve the performance of the mobile terminals is to exploit methods that achieve transmit-diversity. The proposed scheme, denoted as adaptive GSSK (AGSSK), can be thought as a generalization of the method proposed in [23] for two transmit antennas. We analytically show that for the perfect feedback case our solution offers full transmit-diversity for three and four transmit-antennas without reducing the achievable rate.

In addition, due to the fact that the performance of AGSSK degrades as the rate increases due to the smaller average minimum Euclidean distance, we, moreover, propose an enhancing method for AGSSK that relies on the use of time-orthogonal waveforms for the different constellation points. We analytically prove that the transmitdiversity gain achieved by this enhancement is greater than the number of active antennas without any antenna 
selection technique. Monte-Carlo simulations show that the proposed enhanced scheme, which is denoted as AGSSK-TOSD, outperforms for certain MISO configurations, rates, and SNR regions well-known feedback-based multiple-antenna technologies, such as the Equal Gain Transmission (EGT) scheme [27], the antenna subset selection for spatial multiplexing [28], the antenna subset selection for space-time codes [29], and the adaptive SM scheme of [17], as it will be shown in the numerical results of Section 4. Finally, we provide an energy efficiency (EE) comparison of AGSSK-TOSD with the aforementioned multiple-antenna schemes for a target (uncoded) ABEP. Depending on the MISO configuration and the achievable rate AGSSK-TOSD achieves various energy gains over them.

\subsection{Organization}

The rest of the article is organized as follows. In Section 2, the system model and an overview of the GSSK method are presented. In Section 3, the proposed AGSSK and AGSSK-TOSD methods are analyzed. Numerical Results in terms of Monte-Carlo simulation regarding the performance comparison of the proposed AGSSK and AGSSKTOSD methods with GSSK and conventional closed-loop schemes, together with an EE comparison of AGSSKTOSD with those schemes for a target ABEP are given in Section 4. Finally, the conclusions and some ideas for future work are given in Section 5.

\section{System model and background}

\subsection{System model}

We consider a generic MISO communication system with $N_{t}$ transmit antennas. We assume that the receiver uses a Maximum-Likelihood (ML) detector [30], and is able to estimate the $N_{t}$ channel impulse responses perfectly. Consequently, the receiver has perfect CSI. Furthermore, an error-free and zero-delay feedback channel between the receiver and the transmitter is assumed to be available. We consider that the $N_{t} \times 1$ MISO channel matrix $\mathbf{H}$ has entries $h_{i}, i=1,2, \ldots N_{t}$, which can be modeled as independent and identically distributed complex Gaussian random variables with zero-mean and unit-variance (Rayleigh fading).

Notation: The following notation is used throughout this article: (i) $N_{a}$ is the number of RF chains and, consequently, the number of simultaneously active transmitantennas, with $1 \leq N_{a} \leq N_{t}$; (ii) $E_{m}$ is the average total energy transmitted at each time slot by the $N_{a}$ active transmit-antennas. An equal distribution among the active transmit-antennas is assumed, i.e. each active antenna emits a signal with energy $E_{m} / N_{a}$; (iii) $\eta$ is the Additive White Gaussian Noise (AWGN) at the receiver input, with both real and imaginary parts having a power spectral density (PSD) equal to $N_{0} / 2$; (iv) $Q(x)=$ $(1 / \sqrt{2 \pi}) \int_{x}^{\infty} \exp \left(-u^{2} / 2\right) d u$ denotes the $Q$-function; (v) $\gamma=E_{m} / N_{0}$; (vi) (.) denotes the binomial coefficient; (vii) $\lfloor\cdot\rfloor$ denotes the floor function of a real number; and (viii) vectors are denoted in boldface.

\subsection{GSSK modulation overview}

In GSSK modulation, the source generates a random sequence of independent bits, which are grouped in sets of $m=\left\lfloor\log _{2}\left(\begin{array}{l}N_{t} \\ N_{a}\end{array}\right)\right\rfloor$ bits and mapped to one of the possible $N_{H}=2^{m}$ points of the spatial-constellation diagram, which can be chosen randomly or based on the criteria in [8]. Throughout this article, we use the random selection. If the $p$-th spatial-constellation point is chosen, with $p=$ $1,2, \ldots, N_{H}$, then the received signal at a given time-slot is given by:

$$
y_{p}(t)=\left[h_{p(1)} s_{p(1)}(t) h_{p(2)} s_{p(2)}(t) \cdots h_{p\left(N_{\alpha}\right)} s_{p\left(N_{a}\right)}(t)\right] \mathbf{w}_{p}+\eta(t)
$$

where $p(l) \in\left\{1,2, \ldots, N_{t}\right\}, l=1,2, \ldots, N_{a}$, denotes the index of the $l$-th antenna in the $p$-th spatial-constellation point. $s_{p(l)}(t)$ denotes the transmitted symbol of the $p(l)$-th antenna. For GSSK modulation, $s_{p(l)}(t)=$ $\sqrt{E_{m}} u(t) \forall p(l) \in\left\{1,2, \ldots, N_{t}\right\}$ where $u(t)$ denotes the unit-energy pulse waveform for each transmission, i.e., $\int_{-\infty}^{\infty}|u(t)|^{2} d t=1$. $\mathbf{w}_{p}$ is a (complex) $N_{a} \times 1$ vector containing the transmit weights of the $N_{a}$ antennas that belong to the $p$-th spatial-constellation point, under the energy constraint $\left\|\mathbf{w}_{p}\right\|^{2} \leq 1$. In GSSK modulation, no CSI at the transmitter (CSIT) is assumed. Thus, the transmit weights are real numbers, which are designed to provide equal energy allocation, i.e., $w_{p i}=1 / \sqrt{N_{a}}$, for $p=1,2, \ldots, N_{H}$ and $i=1,2, \ldots, N_{a}$. During the detection process, the ML-optimum receiver solves a $N_{H^{-}}$ hypothesis testing problem for the estimation of the index of the $N_{a}$ active antennas. This way, the unique message emitted by the encoder is detected. The achievable rate of GSSK modulation is equal to $R_{\mathrm{GSSK}}=m$.

The Union Bound of the ABEP of GSSK modulation has been computed in [7] for the ML-optimum receiver. By using the tighter Codeword-Union based Bound of ([31], Eq. (35), we have:

$\operatorname{ABEP}_{\mathrm{GSSK}} \leq A \sum_{p}^{N_{H}} \sum_{k, k \neq p}^{N_{H}} N_{b_{p, k}} Q\left(\sqrt{\frac{\gamma}{2 N_{a}}\left|\sum_{l=1}^{N_{a}}\left[h_{p(l)}-h_{k(l)}\right]\right|^{2}}\right)$,

where $A=1 /\left[N_{H} \log _{2}\left(N_{H}\right)\right]$ and $N_{b_{p, k}}$ denotes the number of bits in error between the $p$-th and $k$-th spatialconstellation points, which depends on the GSSK mapper rule [8]. These symbols will also be used in the rest 
of this article. From (2), it is evident that no transmitdiversity can be achieved due to the coherent summation of complex Gaussian channel gains. Furthermore, energy is wasted due to the subtraction of the channel gains of common antennas in different spatial-constellation points.

\section{AGSSK and AGSSK-TOSD}

\subsection{AGSSK}

In AGSSK, we exploit the knowledge of the channel phases to achieve transmit-diversity gains in contrast to GSSK, which we assume to be available at the transmitter through a feedback channel in the case of FrequencyDivision Duplexing (FDD) systems. Its principle is illustrated in Figure 1. By setting the phase of one of the $N_{t}$ wireless links as a reference, denoted as $\phi_{\text {ref }}$, the receiver needs to feedback to the transmitter $N_{t}-1$ phase differences. We denote the phases of the $N_{t}$ channel links by $\phi_{i}, i=1,2, \ldots, N_{t}$. If, for instance, $\phi_{\text {ref }}=\phi_{1}$, the feedback vector $\phi_{\text {feedback }}$ of the phase differences is given by: $\boldsymbol{\phi}_{\text {feedback }}=\left(\begin{array}{lll}\phi_{1}-\varphi_{2} & \cdots & \phi_{1}-\varphi_{N_{t}}\end{array}\right)$. Similar to GSSK modulation, the same pulse waveform $u(t)$ is used for all the constellation points.

\subsubsection{Design of the precoding vectors}

The design of the precoding vectors $\mathbf{w}_{p}, p=1,2, \ldots, N_{H}$ is based on the following observation. From (2), it follows that the main reason why GSSK modulation cannot achieve transmit-diversity gains is the coherent summation of the channel gains, which is caused by the presence of random channel phases in the spatial-constellation points. Consequently, our goal with the proposed precoding design is to avoid random channel phases. Specifically: (i) for any spatial-constellation point, the channel phases of the $N_{a}$ active antennas are aligned to $\phi_{\text {ref, }}$ i.e., they are co-phased. This step is equivalent to conventional Equal Gain Combining (EGC) [30] scheme at the receiver, but it is performed at the transmitter by exploiting the knowledge of $\phi_{\text {feedback; }}$ and (ii) the resulting co-phased $N_{H}$ spatial-constellation points are adequately phase-rotated in order to maximize the minimum Euclidean distance among them, and, consequently, maximize the coding gain of the system. Based on geometrical considerations, this step is equivalent to uniformly distributing the cophased spatial-constellation points over a circle, similar to PSK modulation. Hence, the spatial-constellation diagram resulting after applying the precoding vector geometrically resembles a PSK modulation scheme, whose $N_{H}$ points are random variables given by the summation of $N_{a}$ independent Rayleigh channel gains. Accordingly, the $p$ - th spatial-constellation point, after being phase-aligned, is phase-rotated by $(p-1)\left(2 \pi / N_{H}\right)$ for $p=1,2, \ldots N_{H}$ each time that $\boldsymbol{\phi}_{\text {feedback }}$ is updated.

Why the maximization of the minimum Euclidean distance of the constellation diagram leads to maximization of the coding gain can be understood by considering the well-known nearest neighbor approximation in the high-SNR region to the symbol error probability of a system that employs ML detection ([30], Eq. 5.45). Specifically, the symbol error probability conditioned on the channel matrix $\mathbf{H}$, which we denote as $P_{e}(\mathbf{H})$, can be approximated as $P_{e}(\mathbf{H}) \approx N_{e} Q\left(\sqrt{\frac{\gamma}{2 N_{a}} d_{\min }^{2}(\mathbf{H})}\right)$, where $d_{\min }(\mathbf{H})$ is the minimum Euclidean distance for a particular realization of the channel matrix $\mathbf{H}$ and $N_{e}$ is the average number of neighbors at this distance. Consequently, maximizing $d_{\min }(\mathbf{H})$ leads to the minimization of $P_{e}(\mathbf{H})$ and, hence, to the maximization of the coding gain of the system.

In summary, the $p$-th spatial-constellation point after applying precoding at the transmitter is:

$$
x_{p}(t)=\sqrt{E_{m}}\left(\left|h_{p(1)}\right| e^{j \phi_{p(1)}} \cdots\left|h_{p\left(N_{a}\right)}\right| e^{j \phi_{p\left(N_{a}\right)}}\right) \mathbf{w}_{p} u(t),
$$

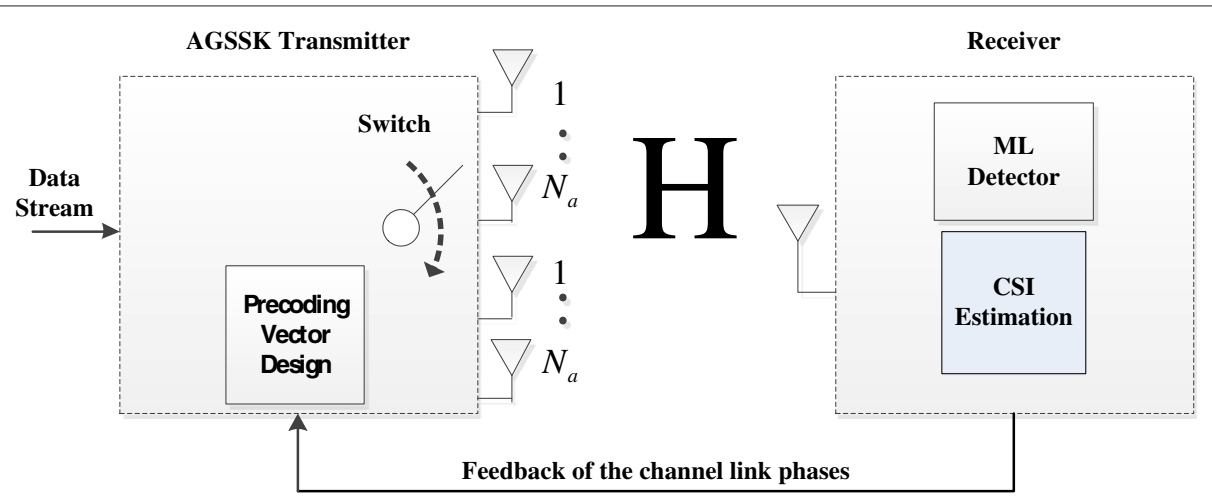

Figure 1 AGSSK principle. 
where $h_{p(l)}=\left|h_{p(l)}\right| e^{j \phi_{p(l)}}$ for $l=1,2, \ldots, N_{a}$, and the precoding vector $\mathbf{w}_{p}$, which takes into account co-phasing and rotation, is:

$$
\mathbf{w}_{p}=\frac{1}{\sqrt{N_{a}}}\left(\begin{array}{c}
e^{j\left(\varphi_{\mathrm{ref}}+(p-1) \frac{2 \pi}{N_{H}}-\varphi_{p(1)}\right)} \\
\vdots \\
e^{j\left(\varphi_{\mathrm{ref}}+(p-1) \frac{2 \pi}{N_{H}}-\varphi_{p\left(N_{a}\right)}\right)}
\end{array}\right)
$$

Consequently, (3) simplifies as follows (dropping the time variable for convenience):

$$
x_{p}=\sqrt{\frac{E_{m}}{N_{a}}}\left(\sum_{l=1}^{N_{a}}\left|h_{p(l)}\right|\right) e^{j\left(\varphi_{\mathrm{ref}}+(p-1) \frac{2 \pi}{N_{H}}\right)}
$$

for $p=1,2, \ldots N_{H}$, which resembles a $N_{H}$-PSK constellation diagram with points being random variables that depend on the channel gains.

At this point, it is important to comment on how the precoding vector design of (4) compares with the precoding vector design of the EGT method of [27], which also uses the channel phases to align the channel links that correspond to the active antennas in order to achieve transmit diversity gains. By observing (4), we see that the channel links that correspond to each constellation point are aligned at a different angle, which is determined by the factor $(p-1)\left(2 \pi / N_{H}\right)$ for $p=1,2, \ldots N_{H}$, so as to obtain a PSK-like spatial constellation diagram and maximize the coding gain. On the other hand, the corresponding precoding vector for EGT MISO systems, which is given by ([27], Eq. (11)) for $N_{r}=1$, is the same for all the constellation points since a conventional signal constellation diagram is used to convey information, in contrast to AGSSK. Hence, the precoding design of (4) for AGSSK includes the additional factor that rotates each constellation point in a way that the coding gain is maximized, compared to the precoding design of EGT, that includes only the phase alignment feature.

\subsubsection{Performance analysis}

By using again ([31], Eq. (35), the ABEP of the proposed adaptive method can be computed as follows:

$$
\mathrm{ABEP}_{\text {Adaptive-GSSK }} \leq A \sum_{p}^{N_{H}} \sum_{k, k \neq p}^{N_{H}} N_{b_{p, k}} \mathrm{PEP}_{x_{p} \rightarrow x_{k} \mid h}
$$

where $\mathrm{PEP}_{x_{p} \rightarrow x_{k} \mid h}$ is the Pairwise Error Probability (PEP) between the spatial-constellation points $x_{p}$ and $x_{k}$, conditioned on the fading paths. This PEP can be computed as follows:

$$
\begin{aligned}
\operatorname{PEP}_{x_{p} \rightarrow x_{k} \mid h} & =Q\left(\sqrt{\frac{1}{2 N_{0}}\left|x_{p}-x_{k}\right|^{2}}\right) \\
& =Q\left(\sqrt{\frac{1}{2 N_{0}}\left[\left|x_{p}\right|^{2}+\left|x_{k}\right|^{2}-2\left|x_{p}\right|\left|x_{k}\right| \cos \left(\phi_{p, k}\right)\right]}\right),
\end{aligned}
$$

where $\phi_{p, k}=|p-k|\left(2 \pi / N_{H}\right) \in(0, \pi]$ is the phase difference between $x_{p}$ and $x_{k}$, which, unlike (2), is no longer a random variable.

By comparing Equation (7) with (2), we notice that the common fading gains between pairs of spatialconstellation points do not cancel out and, furthermore, no random phases are present in the expression of the PEP. This results in transmit-diversity gains and higher coding gains than GSSK, as it will be shown in the numerical results of Section 4.

\subsubsection{Analysis of the diversity order for $N_{t}=3$ and $N_{t}=4$}

Three transmit-antennas: Let us consider a setup with $N_{t}=3$ and $N_{a}=2$, where $N_{H}=2$ and $R=1 \mathrm{bpcu}$. Without loss of generality, we assume that the spatialconstellation diagram is composed by the pairs of antenna indexes given by $(1,2)$ and $(1,3)$, which correspond to the transmission of bits ' 0 ' and ' 1 ', respectively, and $\phi_{\text {ref }}=\phi_{1}$. By using (3) and (4), we have:

$$
\begin{aligned}
x_{1} & =\sqrt{E_{m}}\left(\left|h_{1}\right| e^{j \phi_{1}}\left|h_{2}\right| e^{j \phi_{2}}\right)\left(\begin{array}{l}
1 / \sqrt{2} \\
e^{j\left(\phi_{1}-\phi_{2}\right)} / \sqrt{2}
\end{array}\right) \\
& =\sqrt{\frac{E_{m}}{2}}\left(\left|h_{1}\right|+\left|h_{2}\right|\right) e^{j \phi_{1}}
\end{aligned}
$$

and

$$
\begin{aligned}
x_{2} & =\sqrt{E_{m}}\left(\left|h_{1}\right| e^{j \phi_{1}}\left|h_{3}\right| e^{j \phi_{3}}\right)\left(\begin{array}{l}
e^{j \pi} / \sqrt{2} \\
e^{j\left(\phi_{1}-\phi_{3}+\pi\right)} / \sqrt{2}
\end{array}\right) \\
& =\sqrt{\frac{E_{m}}{2}}\left(\left|h_{1}\right|+\left|h_{3}\right|\right) e^{j\left(\phi_{1}+\pi\right)}
\end{aligned}
$$

By substituting (8) and (9) in (7), we obtain:

$$
\begin{aligned}
\mathrm{PEP}^{\prime} 0^{\prime} \rightarrow 1^{\prime} \mid h & =\mathrm{PEP}^{\prime} 1^{\prime} \rightarrow{ }^{\prime} 0^{\prime} \mid h \\
& =Q\left(\sqrt{\frac{\gamma}{2}}\left[2\left|h_{1}\right|+\left|h_{2}\right|+\left|h_{3}\right|\right]\right)
\end{aligned}
$$

By carefully analyzing (10), we notice that is resembles, except for a scaling factor, the PEP of an EGC system with three receive-antennas. Thus, we can conclude that a diversity order equal to $L=N_{t}=3$ is obtained.

Four transmit-antennas: If $N_{t}=4$, the angle $\phi_{p, k}$ between a pair $\left(x_{p}, x_{k}\right)$ of spatial-constellation points can take two different values: i) $\phi_{p, k}=\pi$; and ii) $\phi_{p, k}=\pi / 2$. Case $\phi_{p, k}=\pi$ : Using (5) and (7), we obtain:

$$
\begin{aligned}
\operatorname{PEP}_{x_{p} \rightarrow x_{k} \mid h} & =Q\left(\sqrt{\frac{\gamma}{N_{a}}}\left[\sum_{l=1}^{N_{a}}\left[\left|h_{p(l)}\right|+\left|h_{k(l)}\right|\right]\right]\right) \\
& =Q\left(\sqrt{\frac{\gamma}{N_{a}}}\left[\sum_{i}^{2 d_{p, k}}\left|h_{i}\right|+\sum_{q, q \neq i}^{N_{a}-d_{p, k}} 2\left|h_{q}\right|\right]\right),
\end{aligned}
$$


where $2 d_{p, k}$ is the number of distinct antenna-indexes between the spatial-constellation points $x_{p}$ and $x_{k}$. Once again, (11) resembles an EGC diversity scheme with $L_{p, k}=$ $2 d_{p, k}+N_{a}-d_{p, k}=N_{a}+d_{p, k}$ antennas at the receiver. Thus, the diversity gain of (11) is $L_{p, k}$.

Case $\phi_{p, k}=\pi / 2$ : By still using (5) and (7), we obtain:

$\operatorname{PEP}_{x_{p} \rightarrow x_{k} \mid h}=Q\left(\sqrt{\frac{\gamma}{N_{a}}\left[\sum_{i}^{2 d_{p, k}}\left|h_{i}\right|^{2}+\sum_{q, q \neq i}^{N_{a}-d_{p, k}} 2\left|h_{q}\right|^{2}+f(h)\right]}\right)$,

where $f(h)=2 \sum_{l=1}^{N_{a}}\left|h_{p}(l)\right| \sum_{m=1}^{N_{a}}\left|h_{k}(m)\right|$. Consequently, $f(h) \geq 0$ for every channel realization. The diversity order $L_{p, k}$ of (12) can be obtained by using the following upper-bound:

$\operatorname{PEP}_{x_{p} \rightarrow x_{k} \mid h} \leq Q\left(\sqrt{\frac{\gamma}{N_{a}}\left[\sum_{i}^{2 d_{p, k}}\left|h_{i}\right|^{2}+\sum_{q, q \neq i}^{N_{a}-d_{p, k}} 2\left|h_{q}\right|^{2}\right]}\right)$,

which stems from the fact that $f(h) \geq 0$ and that the $Q$-function is monotonically decreasing for increasing values of its argument. From the right-side term of (13), we notice that the PEP resembles a Maximum Ratio Combining (MRC) diversity scheme [30] with $L_{p, k}=2 d_{p, k}+N_{a}-d_{p, k}=N_{a}+d_{p, k}$ antennas at the receiver. Thus, the diversity order of (12) is $L_{p, k} \geq$ $N_{a}+d_{p, k}$. If $N_{t}=4$, we conclude that different PEPs might have different diversity gains. Overall, the diversity order $L$ of the system is equal to the minimum of the diversity orders of the PEPs, i.e., $L=\min \left\{L_{p, k}\right\}$ for $p, k=1,2, \ldots, N_{H}$.

Full-Diversity Order $-N_{a}=3$. Let us consider the setup with $N_{a}=3$. In total, there are four combinations of three simultaneously active antennas, i.e., $(1,2,3),(1,2,4)$, $(2,3,4)$, and $(1,3,4)$. Thus, $N_{H}=4$. By direct inspection, we have $2 d_{p, k}=2$ for $p, k=1,2, \ldots, N_{H}$. So, $\left.L\right|_{\phi_{p, k=\pi}}=N_{a}+d_{p, k}=4$ and $\left.L\right|_{\phi_{p, k=\pi / 2}} \geq N_{a}+d_{p, k}=4$. Consequently, $L=N_{t}=4$. Due to the existence of four constellation points, the achievable rate is equal to $R=\log _{2}(4)=2 \mathrm{bpcu}$, which is the maximum achievable rate for $N_{t}=4$.

\subsection{AGSSK-TOSD}

The motivation for introducing AGSSK-TOSD arises by observing (7). The negative term inside the argument of the $Q$-function, which comes as a result of the cross-product between two constellation points, reduces the overall coding and diversity gain. In particular, the higher $N_{H}$ and, consequently, the data rate is, the smaller the angle $\phi_{p, k}$ between two adjacent constellation points is, which increases the value of the cosine inside the argument of the $Q$-function. Hence, the average value of the whole expression inside the argument of the $Q$-function becomes smaller. Consequently, the question that comes into our mind is: could we somehow enhance the AGSSK method by avoiding the negative term inside the argument of the $Q$-function? The answer is affirmative by considering the properties of time-orthogonal waveforms [26]. Specifically, assuming two time-orthogonal waveforms $u_{i}(t)$ and $u_{p}(t)$, then:

$$
\int_{-\infty}^{\infty} u_{i}(t) u_{p}(t) d t= \begin{cases}1 & \text { if } i=p \\ 0 & \text { otherwise }\end{cases}
$$

Based on these properties of time-orthogonal waveforms, our proposal is to apply time-orthogonal pulses to the design of (3). Specifically, the constellation symbols in the time domain are:

$$
x_{p}(t)=\sqrt{\frac{E_{m}}{N_{a}}}\left(\sum_{l=1}^{N_{a}}\left|h_{p(l)}\right|\right) e^{j\left(\varphi_{\mathrm{ref}}+(p-1) \frac{2 \pi}{N_{H}}\right)} u_{p}(t)
$$

for $p=1,2, \ldots N_{H}$. Furthermore:

$$
\left\{\begin{array}{l}
u_{p}(t)=u_{k}(t) \quad \text { if } \quad k=p+\frac{N_{H}}{2} \\
\int_{-\infty}^{\infty} u_{p}(t) u_{k}(t) d t=0 \quad \text { otherwise }
\end{array} p=1,2, \ldots N_{H}\right.
$$

We observe from (16) that the proposed design is a bi-time-orthogonal design that requires a number of timeorthogonal pulses equal to $N_{H} / 2$. This is analogous to the bi-orthogonal signal design ([32], p. 178), which requires half the number of orthogonal signals and has a better ABEP than the full-orthogonal design. In other words, the constellation diagram consists of constellation points with the same pulse and phase difference equal to $\pi$ and points with different pulses that are time-orthogonal with each other.

Example 1. Let us assume that $N_{t}=4$ and $N_{a}=3$. Consequently, there are four possible combinations of three active antennas out of four and so a rate of $2 \mathrm{bpcu}$ is supported. By indexing the four transmit antennas as 1, 2, 3 , and 4 , the four possible combinations of three active antennas are: $(1,2,3),(1,2,4),(1,3,4)$, and $(2,3,4)$. So, the 
four possible waveforms to be transmitted based on (15) and (16) are:

$$
\begin{aligned}
& x_{1}(t)=\sqrt{\frac{E_{m}}{N_{a}}}\left(\left|h_{1}\right|+\left|h_{2}\right|+\left|h_{3}\right|\right) e^{j \varphi_{\mathrm{ref}}} u_{1}(t) \\
& x_{2}(t)=\sqrt{\frac{E_{m}}{N_{a}}}\left(\left|h_{1}\right|+\left|h_{2}\right|+\left|h_{4}\right|\right) e^{j\left(\varphi_{\mathrm{ref}}+\frac{\pi}{2}\right)} u_{2}(t) \\
& \left.x_{3}(t)=\sqrt{\frac{E_{m}}{N_{a}}}\left(\left|h_{1}\right|+\left|h_{3}\right|+\left|h_{4}\right|\right)\right) e^{j\left(\varphi_{\mathrm{ref}}+\pi\right)} u_{1}(t) \\
& x_{4}(t)=\sqrt{\frac{E_{m}}{N_{a}}}\left(\left|h_{2}\right|+\left|h_{3}\right|+\left|h_{4}\right|\right) e^{j\left(\varphi_{\mathrm{ref}}+\frac{3 \pi}{2}\right)} u_{2}(t),
\end{aligned}
$$

where $u_{1}(t)$ and $u_{2}(t)$ are time-orthogonal with each other.

Example 2. Now, let us assume that $N_{t}=5$ and $N_{a}=2$. There are ten possible combinations of two antennas out of five and so we randomly choose eight of them in order to have number of combinations that is a power of two. Hence, a rate of $3 \mathrm{bpcu}$ is supported. By indexing the five transmit antennas as $1,2,3,4$, and 5 , let us assume that the chosen combinations of two active antenna are: $(1,2)$, $(1,3),(1,4),(1,5),(2,3),(2,4),(2,5)$, and $(3,4)$. Based on $(15)$ and (16), the eight possible waveforms to be transmitted are:

$$
\begin{aligned}
& x_{1}(t)=\sqrt{\frac{E_{m}}{N_{a}}}\left(\left|h_{1}\right|+\left|h_{2}\right|\right) e^{j \varphi_{\mathrm{ref}}} u_{1}(t) \\
& x_{2}(t)=\sqrt{\frac{E_{m}}{N_{a}}}\left(\left|h_{1}\right|+\left|h_{3}\right|\right) e^{j\left(\varphi_{\mathrm{ref}}+\frac{\pi}{4}\right)} u_{2}(t) \\
& x_{3}(t)=\sqrt{\frac{E_{m}}{N_{a}}}\left(\left|h_{1}\right|+\left|h_{4}\right|\right) e^{j\left(\varphi_{\mathrm{ref}}+\frac{\pi}{2}\right)} u_{3}(t) \\
& x_{4}(t)=\sqrt{\frac{E_{m}}{N_{a}}}\left(\left|h_{1}\right|+\left|h_{5}\right|\right) e^{j\left(\varphi_{\mathrm{ref}}+\frac{3 \pi}{4}\right)} u_{4}(t) \\
& x_{5}(t)=\sqrt{\frac{E_{m}}{N_{a}}}\left(\left|h_{2}\right|+\left|h_{3}\right|\right) e^{j\left(\varphi_{\mathrm{ref}}+\pi\right)} u_{1}(t) \\
& x_{6}(t)=\sqrt{\frac{E_{m}}{N_{a}}}\left(\left|h_{2}\right|+\left|h_{4}\right|\right) e^{j\left(\varphi_{\mathrm{ref}}+\frac{5 \pi}{4}\right)} u_{2}(t) \\
& x_{7}(t)=\sqrt{\frac{E_{m}}{N_{a}}}\left(\left|h_{2}\right|+\left|h_{5}\right|\right) e^{j\left(\varphi_{\mathrm{ref}}+\frac{3 \pi}{2}\right)} u_{3}(t) \\
& x_{8}(t)=\sqrt{\frac{E_{m}}{N_{a}}}\left(\left|h_{3}\right|+\left|h_{4}\right|\right) e^{j\left(\varphi_{\mathrm{ref}}+\frac{7 \pi}{4}\right)} u_{4}(t),
\end{aligned}
$$

where $u_{1}(t), \quad u_{2}(t), \quad u_{3}(t)$, and $u_{4}(t)$ are pair-wise time-orthogonal.

Let us now provide a discussion regarding the difference between the implementation of time-orthogonal shaping filters in [22,26] and in our AGSSK-TOSD proposal. In [22,26], the time-orthogonal shaping filters are used on an antenna-basis, which means that each transmit antenna use a different shaping filter and all of them are time-orthogonal with respect to each other. On the other hand, in AGSSK-TOSD the time-orthogonal shaping filters are used on a constellation point-basis and not on an antenna basis, according to the bi-time-orthogonal design of (16). As it is proven in the Appendix, this design leads to the elimination of the cross-product between constellation points during the ML detection process and in Section 3.2.2 we prove that a transmit-diversity order that is greater than the number of active transmit antennas is achieved.

At this point, it is also important to provide a discussion regarding the bandwidth requirements of using time-orthogonal shaping filters. Such a discussion has been provided in ([26], Section V), where it is shown that depending on the technology used to design them, time-orthogonal shaping filters can be designed for narrowband systems with the desired bandwidth spectrum. As it is stated, the method that is proposed in [33] for the design of the timeorthogonal shaping filters guarantees that all of them have the same time-duration and (practical) bandwidth. Although the time-orthogonal filter design in [33] was proposed for Ultra Wide Band systems, the authors show in [26] that the time-duration and bandwidth of the filters can be adequately scaled for narrowband systems, and so the same design can be used. Hence, the time-orthogonal shaping filters can be designed to have the same bandwidth requirements as a single filter narrowband system.

\subsubsection{Performance analysis}

Let us assume that $x_{p}$ and $x_{k}$ (dropping the time variable for convenience), $p, k=1,2, \ldots N_{H}$, are two possible constellation symbols to be transmitted, which are given by (15). By using again ([31], Eq. (35), the ABEP of AGSSKTOSD is computed as

$$
\mathrm{ABEP}_{\text {AGSSK-TOSD }} \leq A \sum_{p}^{N_{H}} \sum_{k, k \neq p}^{N_{H}} N_{b_{p, k}} \mathrm{PEP}_{x_{p} \rightarrow x_{k} \mid h}
$$

Considering the design of (16), the PEP conditioned on the instantaneous channel realization is given by (see the Appendix for the proof): 


$$
\operatorname{PEP}_{x_{p} \rightarrow x_{k} \mid h}= \begin{cases}Q\left(\sqrt{\frac{\gamma}{2 N_{a}}}\left[\sum_{l=1}^{N_{a}}\left(\left|h_{p(l)}\right|+\left|h_{k(l)}\right|\right)\right]\right) & \text { if } k=p+\frac{N_{H}}{2} \\ Q\left(\sqrt{\left.\frac{\gamma}{2 N_{a}}\left[\left(\sum_{l=1}^{N_{a}}\left|h_{p(l)}\right|\right)^{2}+\left(\sum_{l=1}^{N_{a}}\left|h_{k(l)}\right|\right)^{2}\right]\right)}\right) & \text { otherwise }\end{cases}
$$

(20) can be also written as:

$$
\mathrm{PEP}_{x_{p} \rightarrow x_{k} \mid h}= \begin{cases}Q\left(\sqrt{\frac{\gamma}{2 N_{a}}}\left[\sum_{i}^{2 d_{p, k}}\left|h_{i}\right|+\sum_{q, q \neq i}^{N_{a}-d_{p, k}} 2\left|h_{q}\right|\right]\right) & \text { if } k=p+\frac{N_{H}}{2} \\ Q\left(\sqrt{\frac{\gamma}{2 N_{a}}\left[\sum_{i}^{2 d_{p, k}}\left|h_{i}\right|^{2}+\sum_{q, q \neq i}^{N_{a}-d_{p, k}} 2\left|h_{q}\right|^{2}+f(h)\right]}\right) \text { otherwise, }\end{cases}
$$

where again $2 d_{p, k}$ is the number of distinct antenna indices between the constellation points $x_{p}$ and $x_{k}$, and $f(h)=2 \sum_{l=1}^{N_{a}}\left|h_{p}(l)\right| \sum_{m=1}^{N_{a}}\left|h_{k}(m)\right|$, as in Section 3.1.2 regarding the AGSSK concept. Hence, $f(h) \geqslant 0$ for every channel realization. From (20), we clearly see the advantage of applying time-orthogonal pulses in the AGSSK design: The cross product between the constellation points is eliminated and what remains is the summation of the squared amplitudes of the points.

\subsubsection{Analysis of the diversity order}

Case $k=p+\frac{N_{H}}{2}$ :

By direct inspection of (21), we observe that the argument inside the $Q$-function of the PEP is a summation of independent Rayleigh variables. This is similar to the EGC scheme, and so we conclude that the diversity order is equal to $2 d_{p, k}+N_{a}-d_{p, k}=N_{a}+d_{p, k}$.

Case $k \neq p+\frac{N_{H}}{2}$ :

By observing (21), the argument inside the $Q$ function consists of the summation of independent Chi-squared distributed variables, plus a positive term. Consequently,

$$
\begin{gathered}
Q\left(\sqrt{\frac{\gamma}{2 N_{a}}\left[\sum_{i}^{2 d_{k, p}}\left|h_{i}\right|^{2}+\sum_{q, q \neq i}^{N_{a}-d_{k, p}} 2\left|h_{q}\right|^{2}+f(h)\right]}\right) \\
\leqslant Q\left(\sqrt{\frac{\gamma}{2 N_{a}}\left[\sum_{i}^{2 d_{k, p}}\left|h_{i}\right|^{2}+\sum_{q, q \neq i}^{N_{a}-d_{k, p}} 2\left|h_{q}\right|^{2}\right]}\right) .
\end{gathered}
$$

The diversity order of the right-hand term in (22) is equal to $N_{a}+d_{p, k}$, due to its MRC nature. Hence, the diversity order of the left-hand term of (22) is larger or equal than $N_{a}+d_{p, k}$. As a result, the total diversity of the system is equal to $N_{a}+\min \left\{d_{p, k}\right\}$, which is greater than the number of active transmit antennas $N_{a}$.

\subsection{Applicability of similar to AGSSK phase precoding methods to SM systems}

The aim of this section is to provide a discussion regarding the possibility of applying similar phase precoding methods, as in AGSSK, to indicative SM schemes that incorporate complex constellation signal diagrams apart from the spatial one. One scheme that comes naturally as a scheme to consider is the GSM scheme of [6], which is the SM version of [8] in which the information is carried by both the antennas that are activated for transmission and the complex symbols that are emitted from them. In the general case of a QAM constellation symbol, again the estimates of the channel phases at the receiver can be fed to the transmitter and used to align the channels that correspond to the antennas that are activated for transmission and achieve transmitdiversity. However, in contrast to the precoding design of (4) in which the constellation points of GSSK are uniformly distributed over a circle, the positioning of the constellation symbols in a precoded GSM that will maximize the coding gain would result in an optimization problem that the receiver should solve each time that it needs to feed the phases to the transmitter. Consequently, this would lead to a higher computational requirements at the receiver than the precoded GSSK since the positions of the phase aligned constellation symbols need to be calculated at the receiver in the general case of QAM symbols.

As far as the expected diversity gain of such a precoded GSM method is concerned, it is expected to be no higher than the number of active transmit antennas regardless of using or not time-orthogonal waveforms. 
This is due to the fact that the constellation diagram would consist of some pairs of points which differ only in the complex signal symbol and not in the the channel links of the spatial constellation diagram. This would be a disadvantage compared to AGSSK-TOSD, for instance, for which we proved that the minimum diversity order is greater than the number of active antennas. However, an advantage with respect to the AGSSK/AGSSKTOSD design would be the capability of achieving the same rates with a smaller total number of transmit antennas.

As far as other SM-based schemes are concerned, two interesting concepts for considering the possibility of applying precoding is the generalized STSK concept of [14] and the multistream SM idea of [34]. Although the exact phase precoding design of (4) is suitable only for GSSK systems, a different closed-loop phase precoding design could be applied to [14,34], with the possibility of transmit-diversity gains. In particular, based on the CSI estimation at the receiver, the receiver can solve an optimization problem, each time that the channel changes, in order to calculate the phase precoding values for each of the transmit antennas that would result in the lowest instantaneous Bit Error Rate, for instance. This phase values could then be dispatched form the receiver to the transmitter through a low-delay feedback channel. The drawback of this implementation is the exhaustive search that would be required at the receiver, which would pose serious applicability problems. An alleviation of this complexity could be, for instance, realized by the existence of a number of common phase precoding vectors that are known to both the transmitter and the receiver. Hence, the receiver would choose the precoding vector that results in the smallest instantaneous Bit Error Rate and feed the vector's corresponding index to the transmitter.

\section{Numerical results}

The aim of this section is twofold: (i) To provide a performance comparison by means of Monte-Carlo simulations of our proposed AGSSK and AGSSK-TOSD schemes with the open-loop GSSK modulation [7] and also with the well-known closed-loop and transmit diversity achieving multiple antenna schemes of EGT [27], antenna subset selection for spatial multiplexing [28], antenna subset selection for space-time codes [29], and the adaptive SM method of [17] for various MISO setups and achievable rates. For EGT, we consider the selection of the best subset of $N_{a}$ antennas (that results in the highest SNR) at the receiver, which exploits all the available transmit antennas and offers full transmit-diversity gains, and the random selection, which offers transmit-diversity gains equal to the number of active transmit antennas. The random selection can be applicable to low-complexity terminals where the selection of the best subset would pose significant hardware challenges. Moreover, for the antenna subset selection for spatial multiplexing and space-time codes methods we consider the minimum number of RF chains that are needed for these schemes to work, i.e. number of RF chains equal to two. In order to substantiate our diversity analysis, in the figures we also show the Union Bound analytical models in (6) and (19) for AGSSK and AGSSK-TOSD, respectively. More specifically, the curves have been obtained by calculating (6) and (19) for many channel realizations and numerically computing the average. Regarding the generation of time-orthogonal shaping filters for AGSSK-TOSD with the same time duration and bandwidth, we consider the method described in [26]. (ii) To also provide an EE comparison for a target (uncoded) ABEP, regarding the required energy at the transmitter, of AGSSK-TOSD with the aforementioned closed-loop multiple-antenna transmission methods.

\subsection{Performance comparison}

Figure 2 illustrates the performance comparison of AGSSK and AGSSK-TOSD with closed-loop schemes and the open-loop GSSK and GSSK for $N_{t}=3, N_{a}=2$, and a rate of 1 bpcu. Among the possible $\left(\begin{array}{l}3 \\ 2\end{array}\right)=3$ subsets of 2 antennas, we randomly select two of them for the AGSSK proposals and so a rate of 1 bpcu can be supported. As we observe, AGSSK and AGSSK-TOSD significantly outperform GSSK, as expected, and also EGT with random subset selection and closed-loop Alamouti. However, they are outperformed by EGT with selection of the best subset of antennas for all the SNR range. Moreover, the Union Bound model of (6) and (19) for AGSSK and AGSSK-TOSD, respectively, perfectly matches the Monte Carlo simulations in the high-SNR region, as expected.

Figure 3 illustrates the performance comparison of AGSSK and AGSSK-TOSD with closed-loop schemes and the open-loop GSSK for $N_{t}=4, N_{a}=2$, and a rate of 2 bpcu. Among the possible $\left(\begin{array}{l}4 \\ 2\end{array}\right)=6$ subset of 2 antennas, we randomly select four of them for the AGSSK proposals and so a rate of $2 \mathrm{bpcu}$ can be supported. As we observe, the trends are the same as in the case of $1 \mathrm{bpcu}$ rate, i.e. AGSSK and AGSSK-TOSD outperform all schemes except for EGT with selection of the best subset of antennas. Furthermore, again the Union Bound model of (6) and (19) perfectly matches the Monte-Carlo simulations in the high-SNR region.

Figure 4 shows the performance comparison of AGSSK and AGSSK-TOSD with closed-loop schemes and the open-loop GSSK for a rate of $3 \mathrm{bpcu}$, where the $N_{t}=6$, $N_{a}=4$ configuration was chosen. Consequently, among the possible $\left(\begin{array}{l}6 \\ 4\end{array}\right)=15$ subsets, eight are chosen randomly to support a rate of $3 \mathrm{bpcu}$. As we see in this case, 



Figure 3 Performance comparison of AGSSK and AGSSK-TOSD with closed-loop schemes and the open-loop GSSK for $\mathbf{N}_{t}=4, \mathbf{N}_{a}=2$, and a rate of $2 \mathrm{bpcu}$. 


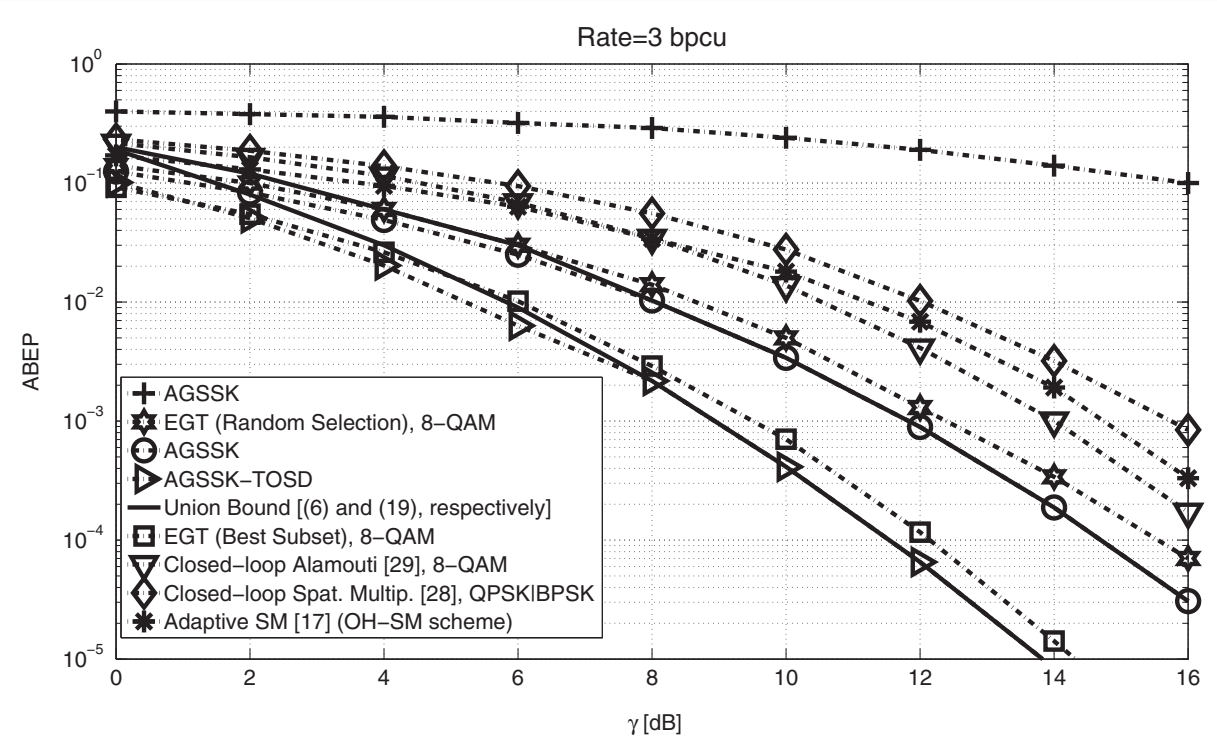

Figure 4 Performance comparison of AGSSK and AGSSK-TOSD with closed-loop schemes and the open-loop GSSK for $\mathbf{N}_{t}=6, \mathbf{N}_{a}=4$, and a rate of $3 \mathrm{bpcu}$.

AGSSK-TOSD outperforms EGT with selection of the best subset of antennas and significantly the other closedloop schemes for the examined SNR range and up to an ABEP value of $10^{-5}$. Due to its full transmit diversity, EGT with selection of the best subset of antennas and closedloop Alamouti will start outperforming AGSSK-TOSD at some ABEP point, but as we see this point occurs for an $A B E P$ value smaller than $10^{-5}$, which is much lower than the uncoded ABEP requirements of many practical systems.

Now, Figure 5 illustrates the comparison of AGSSK and AGSSK-TOSD with closed-loop schemes and the openloop GSSK for a rate of $4 \mathrm{bpcu}$. We chose the configuration $N_{t}=6, N_{a}=3$ to achieve such a rate for the AGSSK proposals. As we observe, up to an ABEP of $10^{-5}$, AGSSK-TOSD and EGT with selection of the

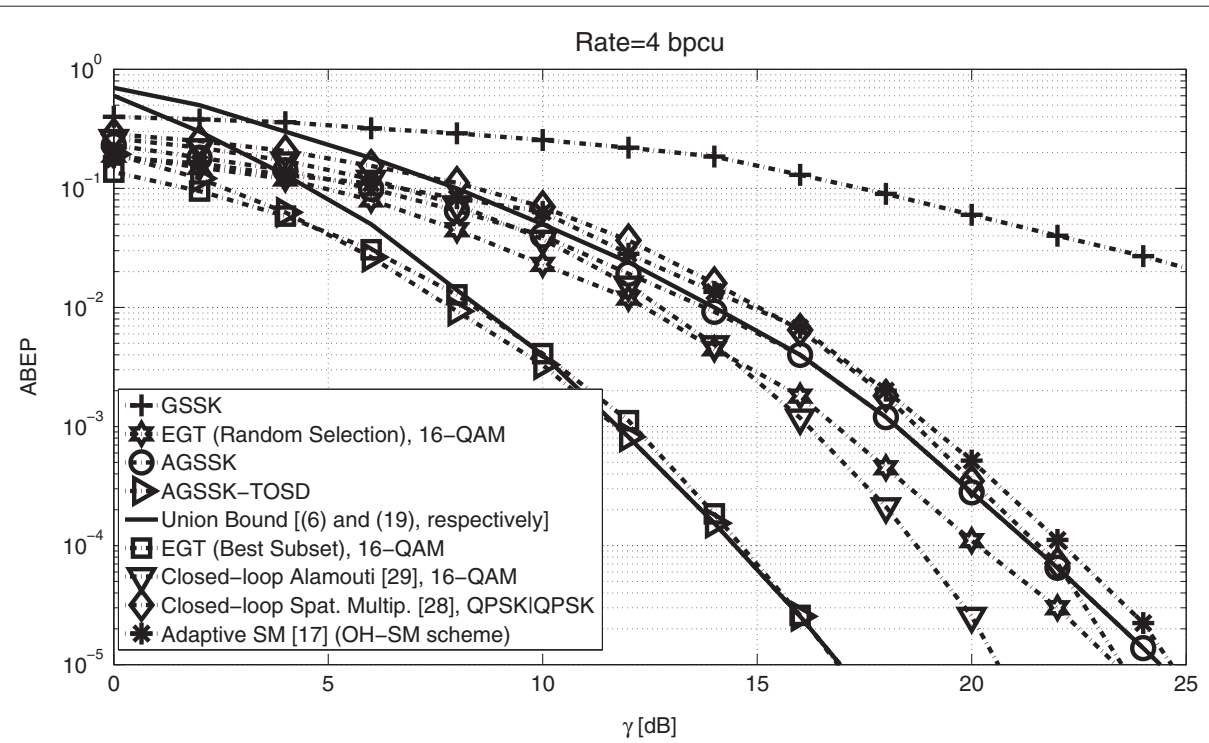

Figure 5 Performance comparison of AGSSK and AGSSK-TOSD with closed-loop schemes and the open-loop GSSK for $\mathbf{N}_{t}=6, \mathbf{N}_{a}=3$, and a rate of $4 \mathrm{bpcu}$. 


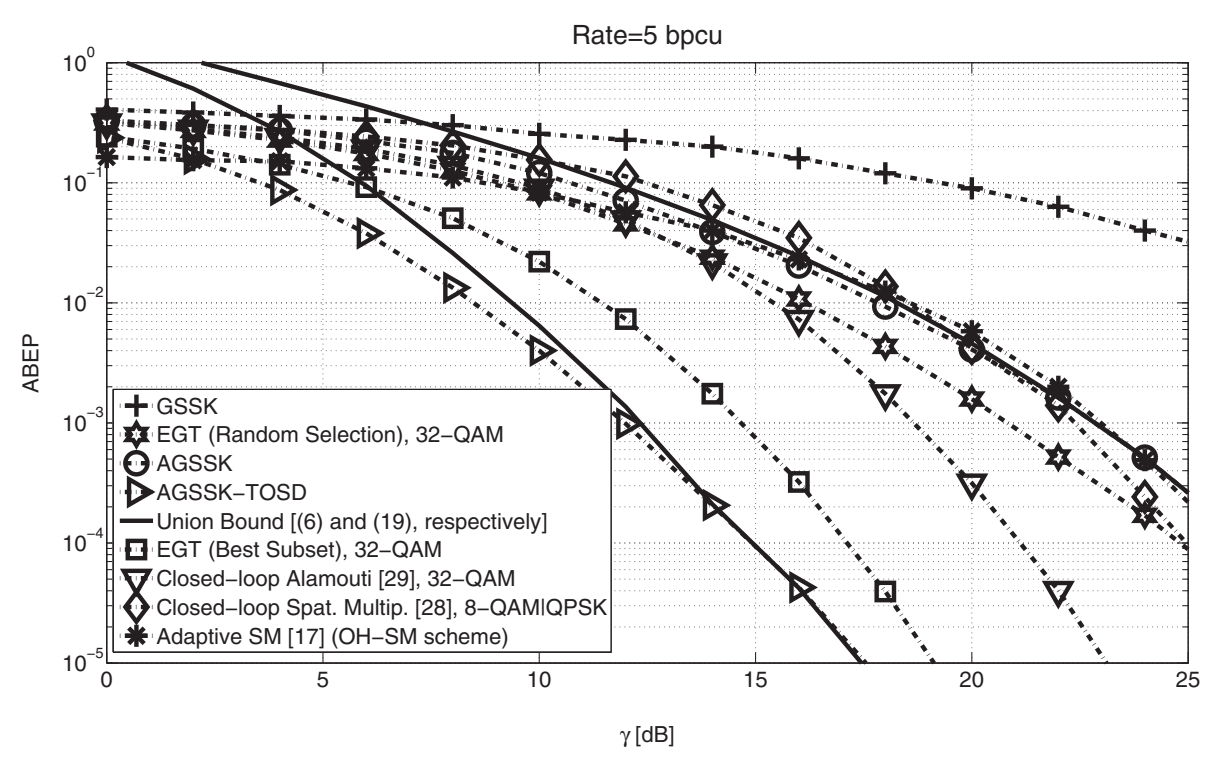

Figure 6 Performance comparison of AGSSK and AGSSK-TOSD with closed-loop schemes and the open-loop GSSK for $\mathbf{N}_{t}=7, \mathbf{N}_{a}=3$, and a rate of $5 \mathrm{bpcu}$.

best subset of antennas have almost the same performance. However, AGSSK-TOSD would be the preferable choice because it avoids any kind of conventional modulation order in contrast to EGT and so the RF amplifier avoids the stringent linearity requirements of the RF amplifiers designed for QAM. In addition, it significantly outperforms the other schemes for the examined SNR range.
Figure 6 shows how the methods compare in terms of performance for a rate of $5 \mathrm{bpcu}$. The configuration $N_{t}=$ $7, N_{a}=3$ is used for achieving such a rate for the AGSSK proposals. As we observe, AGSSK-TOSD significantly outperform all the other schemes in this case. An intuitive reason for this is that the minimum Euclidean distance of QAM gets smaller as the rate and, consequently, the size of the constellation diagram increases. This is a clear

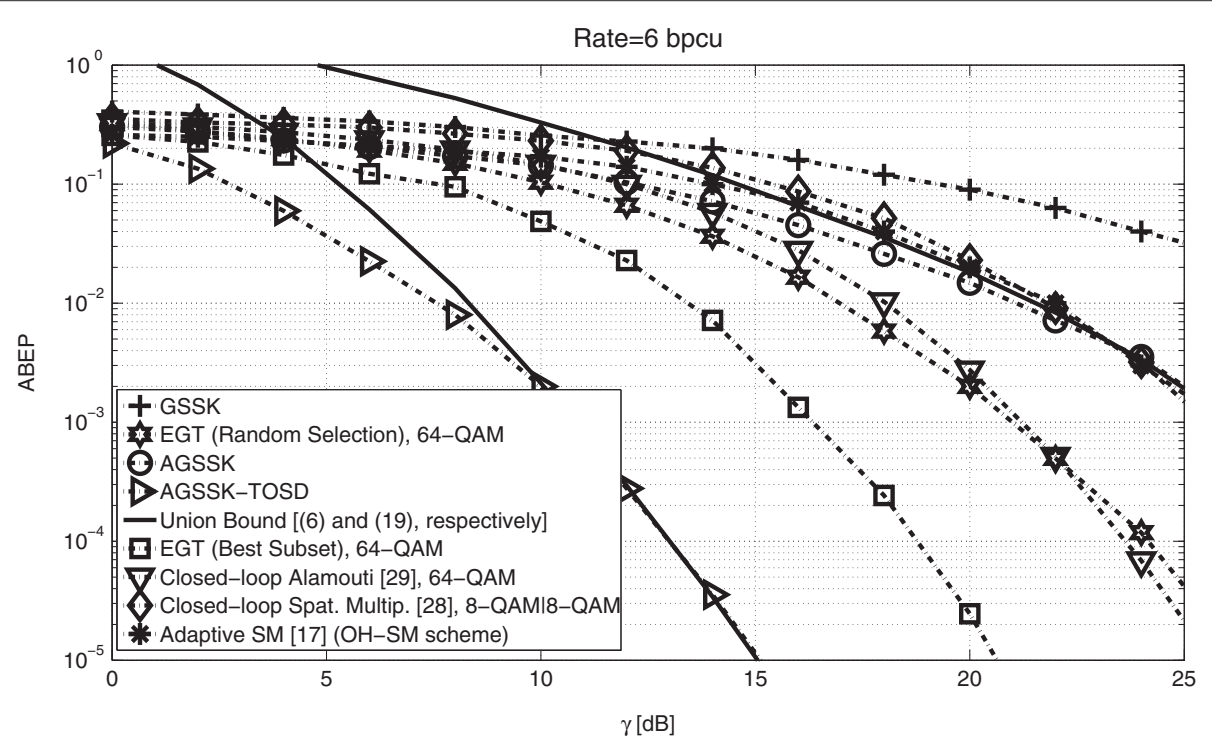

Figure 7 Performance comparison of AGSSK and AGSSK-TOSD with closed-loop schemes and the open-loop GSSK for $\mathbf{N}_{t}=8, \mathbf{N}_{a}=4$, and a rate of 6 bpcu. 
Table 1 Relative EE gain of AGSSK-TOSD over other closed-loop schemes for a target (uncoded) ABEP of $10^{-3}$

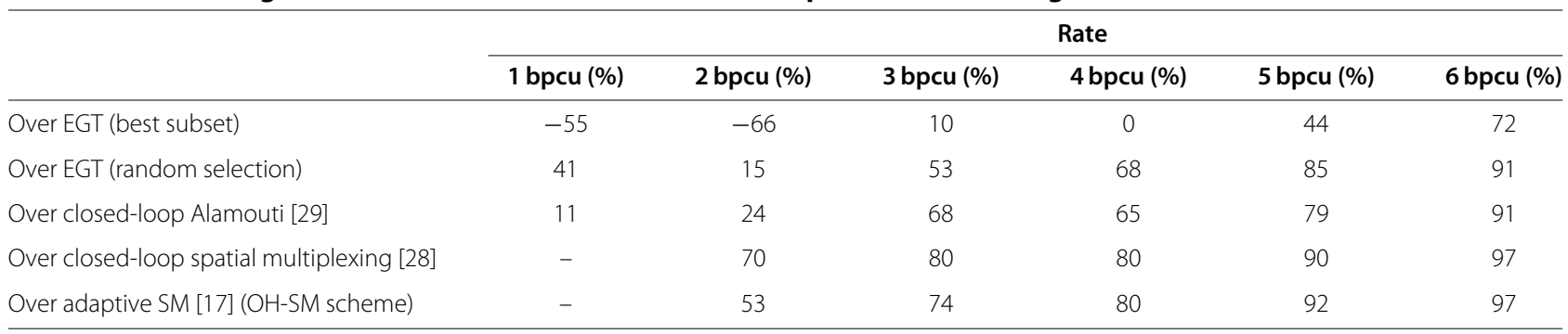

indication that the proposed scheme is useful for medium to high rates.

Finally, Figure 7 shows how the methods compare in terms of performance for a rate of $6 \mathrm{bpcu}$. The configuration $N_{t}=8, N_{a}=4$ achieves such a rate for the AGSSK proposals. As in the case of a rate of $5 \mathrm{bpcu}$, AGSSK-TOSD significantly outperforms all the examined schemes.

\subsection{EE comparison}

We will now provide an EE comparison regarding the energy spent for transmission between AGSSK-TOSD, which is the best scheme in terms of performance with respect to AGSSK and the examined closed-loop multiple-antenna schemes for the MISO configurations of Section 4.1 and for a target ABEP of $10^{-3}$. In particular, we are interested in the relative EE gain, which is calculated as

$$
\text { relative } \mathrm{EE}=\frac{\gamma_{\text {conventional scheme }}-\gamma_{\text {AGSSK }}-\mathrm{TOSD}}{\gamma_{\text {conventional }} \text { scheme }} \times 100 \%
$$

The respective values are given in Table 1.

As we see from Table 1, AGSSK-TOSD can be significantly more energy efficient than the conventional schemes as the rate increases. This is due to the fact that the minimum Euclidean distance for the conventional schemes gets smaller as the rate and, consequently, the QAM order increases, something which is avoided in the AGSSK-TOSD scheme due to the elimination of the cross product between constellation points in the detection process. Hence, although some of the baseline closed-loop schemes, such as EGT and space-time codes with selection of the best subset of antennas, achieve higher transmit-diversity gains than AGSSK-TOSD, there are SNR regions where the latter proposed scheme outperforms them due to its higher coding gain.

\section{Conclusions}

In this article, we introduced the AGSSK and AGSSKTOSD precoding methods for MISO systems, which significantly improve the performance of the conventional GSSK modulation method due to the achieved transmit-diversity and higher coding gains. In addition, the use of time-orthogonal shaping filters for AGSSKTOSD enables diversity gains larger than the number of active antennas without any antenna selection technique, as it was proven. We, moreover, compared by means of Monte Carlo simulations our proposed methods with the conventional open loop GSSK and well-known closed-loop methods for the same number of transmit antennas in terms of performance and in terms of the relative $\mathrm{EE}$ gain for a target $\mathrm{ABEP}$. Results show that for an adequate number of active antennas and for medium to high-rates AGSSK-TOSD, is the preferred transmission technique for the examined scenarios.

Future work will consider the impact of quantized feedback and imperfect channel estimation at the receiver on the performance and EE comparison between AGSSK-TOSD and the examined closed-loop schemes.

\section{Appendix}

Let us consider two constellation points, $x_{p}(t)$ and $x_{k}(t)$, $p, k=1,2, \ldots, N_{H}$, of the proposed AGSSK-TOSD concept. According to (15), $x_{p}(t)=\sqrt{\frac{E_{m}}{N_{a}}}\left(\sum_{l=1}^{N_{a}}\left|h_{p(l)}\right|\right)$ $e^{j \phi_{p}} u_{p}(t)$ and $x_{k}(t)=\sqrt{\frac{E_{m}}{N_{a}}}\left(\sum_{l=1}^{N_{a}}\left|h_{k(l)}\right|\right) e^{j \phi_{k}} u_{k}(t)$. Now, let as assume that the point $x_{p}(t)$ is conveyed, which means that $y(t)=x_{p}(t)+n(t)$. The decision metric is given by (35)

$D_{i}=\operatorname{Re}\left\{\int_{0}^{T_{m}} y(t) x_{i}^{*}(t) d t\right\}-\frac{1}{2} \int_{0}^{T_{m}} x_{i}(t) x_{i}^{*}(t) d t, i=p, k$

where $T_{m}$ is the symbol duration. Consequently, the probability that the $p_{\text {th }}$ constellation point is detected instead of $k_{\mathrm{th}}$, conditioned on the channel statistics, is given by 


$$
\begin{aligned}
& \operatorname{Pr}_{r}\left(D_{p}<D_{k} \mid h\right)= \\
& =\operatorname{Pr}\left(\begin{array}{l}
\frac{1}{2} \frac{E_{m}}{N_{a}}\left(\sum_{l=1}^{N_{a}}\left|h_{p(l)}\right|\right)^{2}+\frac{1}{2} \frac{E_{m}}{N_{a}}\left(\sum_{l=1}^{N_{a}}\left|h_{k(l)}\right|\right)^{2}+\sqrt{\frac{E_{m}}{N_{a}}} \sum_{l=1}^{N_{a}}\left|h_{p(l)}\right| \operatorname{Re}\left\{\int_{0}^{T_{m}} n(t) e^{-j \phi_{p}} u_{p}^{*}(t) d t\right\} \\
\left.<\frac{E_{m}}{N_{a}} \sum_{l=1}^{N_{a}}\left|h_{p(l)}\right| \sum_{l=1}^{N_{a}}\left|h_{k(l)}\right| e^{j \phi_{p}} e^{-j \phi_{k}} \int_{0}^{T_{m}} u_{p}(t) u_{k}^{*}(t) d t+\sqrt{\frac{E_{m}}{N_{a}}} \sum_{l=1}^{N_{a}}\left|h_{k(l)}\right| \operatorname{Re}\left\{\int_{0}^{T_{m}} n(t) e^{-j \phi_{k}} u_{k}^{*}(t) d t\right\}\right)
\end{array}\right.
\end{aligned}
$$

Case 1. $k=p+\frac{N_{H}}{2}$. Then, $\phi_{k}=\pi+\phi_{p}$ according to the bi-time-orthogonal design of (16). Consequently, from (24) we get

$$
P_{r}\left(D_{p}<D_{k} \mid h\right)=Q\left(\sqrt{\frac{\gamma}{2 N_{a}}}\left[\sum_{l=1}^{N_{a}}\left[\left|h_{p(l)}\right|+\left|h_{k(l)}\right|\right]\right]\right)
$$

Case 2. $k \neq p+\frac{N_{H}}{2}$. Then, $u_{p}(t)$ and $u_{k}(t)$ are timeorthogonal and so we get from (24)

$$
\begin{aligned}
& P_{r}\left(D_{p}<D_{k} \mid h\right)= \\
& =P_{r}\left(\begin{array}{l}
\frac{1}{2} \frac{E_{m}}{N_{a}}\left(\sum_{l=1}^{N_{a}}\left|h_{p(l)}\right|\right)^{2}+\frac{1}{2} \frac{E_{m}}{N_{a}}\left(\sum_{l=1}^{N_{a}}\left|h_{k(l)}\right|\right)^{2} \\
<\sqrt{\frac{E_{m}}{N_{a}}} \sum_{l=1}^{N_{a}}\left|h_{k(l)}\right| \operatorname{Re}\left\{\int_{0}^{T_{m}} n(t) e^{-j \phi_{k}} u_{k}^{*}(t) d t\right\} \\
-\sqrt{\frac{E_{m}}{N_{a}}} \sum_{l=1}^{N_{a}}\left|h_{p(l)}\right| \operatorname{Re}\left\{\int_{0}^{T_{m}} n(t) e^{-j \phi_{p}} u_{p}^{*}(t) d t\right\}
\end{array}\right) \\
& =Q\left(\sqrt{\frac{\gamma}{2 N_{a}}\left[\left(\sum_{l=1}^{N_{a}}\left|h_{p(l)}\right|\right)^{2}+\left(\sum_{l=1}^{N_{a}}\left|h_{k(l)}\right|\right)^{2}\right]}\right)
\end{aligned}
$$

which is due to the fact that the factors $\operatorname{Re}\left\{\int_{0}^{T_{m}} n(t) e^{-j \phi_{p}} u_{p}^{*}(t) d t\right\}$ and $\operatorname{Re}\left\{\int_{0}^{T_{m}} n(t) e^{-j \phi_{k}} u_{k}^{*}(t) d t\right\}$ are independent zero-mean normally distributed processes with variance equal to $N_{0} / 2$ each. The uncorrelatedness (and, consequently, independence) between them is due to the orthogonality of $u_{p}(t)$ and $u_{k}(t)$ ([32], p. 234).

\section{Competing interests}

The authors declare that they have no competing interests.

\section{Acknowledgments}

The research leading to these results has received funding from the Spanish Ministry of Science and Innovation (Ministerio de Ciencia e Innovacion) under the project GRE3NLINK-MAC (TEC2011-29006-C03-02), the Catalan Government (2009SGR0891), and the research projects: CO2GREEN (TEC2010-20823), GREENET (PITN-GA-2010-264759), and GREEN-T (CP8-006).

\section{Author details}

${ }^{1}$ Telecommunications Technological Centre of Catalonia (CTTC), Castelldefels, Spain. ${ }^{2}$ L2S, UMR 8506 CNRS - SUPELEC - Univ Paris-Sud, Laboratory of Signals and Systems (L2S), French National Center for Scientific Research (CNRS), École Supérieure d'Électricité (SUPÉLEC), University of Paris-Sud XI (UPS), 3 rue Joliot-Curie, 91192 Gif-sur-Yvette (Paris), France.

Received: 26 November 2012 Accepted: 25 January 2013 Published: 21 February 2013

\section{References}

1. RY Mesleh, H Haas, S Sinanović, CW Ahn, S Yun, Spatial modulation. IEEE Trans. Veh. Technol. 57, 2228-2241 (2008)

2. J Jeganathan, A Ghrayeb, L Szczecinski, Spatial modulation: optimal detection and performance analysis. IEEE Commun. Lett. 12, 545-547 (2008)

3. MD Renzo, H Haas, PM Grant, Spatial modulation for multiple-antenna wireless systems: a survey. IEEE Commun. Mag. 49, 182-191 (2011)

4. P Wolniansky, G Foschini, G Golden, R Valenzuela, in Proceedings of URSI ISSSE. V-BLAST: An architecture for realizing very high data rates over the rich scattering wireless channel, Pisa, Italy, 1998), pp. 295-300

5. MD Renzo, H Haas, Bit error probability of spatial modulation (SM-) MIMO over generalised fading channels. IEEE Trans. Veh. Tech. 61, 1124-1144 (2012)

6. A Younis, N Serafimovski, R Mesleh, H Haas, in Proceedings of IEEE ASILOMAR Conference, Asilomar Grounds. Generalised spatial modulation, USA, 2010), pp. 1498-1502

7. J Jeganathan, A Ghrayeb, L Szczecinski, A Ceron, Space shift keying modulation for MIMO channels. IEEE Trans. Wirel. Commun. 8, 3692-3703 (2009)

8. J Jeganathan, A Ghrayeb, L Szczecinski, in Proceedings of IEEE Personal, Indoor and Mobile Radio Communications. Generalized space shift keying modulation for MIMO channels, Cannes, France, 2008), pp. 1-5

9. MD Renzo, H Haas, Bit error probability of space modulation over Nakagami-m fading: asymptotic analysis. IEEE Commun. Lett. 15, 1026-1028 (2011)

10. T Handte, A Muller, J Speidel, in Proceedings of IEEE Vehicular Technology Conference-Fall. BER analysis and optimization of generalized spatial modulation in correlated fading channels, Anchorage, USA, 2009), pp. 1-5

11. MD Renzo, H Haas, in Proceedings of IEEE International Conference on Communications. Transmit-diversity for spatial modulation (SM): towards the design of high-rate spatially-modulated space-time block codes, Kyoto, Japan, 2011), pp. 1-6

12. Ü E Başar, E Aygölü, HV Panayirci, Poor, Space-time block coded spatial modulation. IEEE Trans. Commun. 59, 823-832 (2011)

13. S Sugiura, S Chen, L Hanzo, Coherent and differential space-time shift keying: a dispersion matrix approach. IEEE Trans. Commun. 58, 3219-3230 (2010)

14. S Sugiura, S Chen, L Hanzo, Generalized spacetime shift keying designed for flexible diversity, multiplexing and complexitytradeoffs. IEEE Trans. Wirel. Commun. 10, 1144-1153 (2011)

15. R Rajashekar, KVS Hari, Modulation diversity for spatial modulation using complex interleaved orthogonal design, arXiv:1204.4073v1, [Online]. Available: http://arxiv.org/abs/1204.4073v1

16. P Yang, Y Xiao, Y Yu, S Li, Adaptive spatial modulation for wireless mimo transmission systems. IEEE Commun. Lett. 15, 602-604 (2011)

17. P Yang, Y Xiao, L Li, Q Tang, Y Yu, S Li, Link adaptation for spatial modulation with limited feedback. IEEE Trans. Veh. Tech. 61, 3808-3813 (2012)

18. WH Chung, CY Hung, in Proceedings of IEEE Vehicular Technology Conference-Spring. Multi-antenna selection using space shift keying in MIMO systems, Yokohama, Japan, 2012), pp. 1-5

19. N Ma, A Wang, C Han, Y Ji, in Proceedings of IEEE International Conference on Communications in China. Adaptive joint mapping generalised spatial modulation, Beijing, China, 2012), pp. 520-523

20. Renzo Di M, H Haas, in Proceedings of IEEE International Conference on Communications. Performance comparison of different spatial modulation schemes in correlated fading channels, Cape Town, South Africa, 2012), pp. 1-6 
21. MD Renzo, H Haas, SSK-MIMO over correlated Rician fading channels: performance analysis and a new method to achieve transmit-diversity gains. IEEE Trans. Commun. 59, 116-129 (2011)

22. MD Renzo, $\mathrm{H}$ Haas, in Proceedings of IEEE International Conference on Communications. Space shift keying (SSK) modulation: on the transmit-diversity/multiplexing trade-off, Kyoto, Japan, 2011)

23. D Yang, L Yang, L Hanzo, Transmit-diversity-assisted space-shift keying for co-located and distributed/cooperative MIMO element. IEEE Trans. Veh. Technol. 60, 2864-2869 (2011)

24. VTarokh, H Jafarkhani, AR Calderbank, Space-time block codes from orthogonal designs. IEEE Trans. Inf. Theory. 45, 1456-1467 (1999)

25. MZA Khan, BS Rajan, Single symbol maximum likelihood decodable linear STBCs. IEEE Trans. Inf. Theory. 52, 2062-2091 (2006)

26. MD Renzo, DD Leonardis, F Graziosi, H Haas, Space shift keying (SSK) MIMO with practical channel estimates. IEEE Trans. Commun. 6, 998-1012 (2012)

27. DJ Love, RW Heath, Equal gain transmission in multiple-input multiple-output wireless systems. IEEE Trans. Commun. 51, 1102-1110 (2003)

28. RW Heath, A Paulraj, in Proceedings of IEEE International Conference on Communications. Antenna selection for spatial multiplexing systems based on minimum error rate, Helsinki, Finland, 2001), pp. 2276-2780

29. AD Gore, A Paulraj, MIMO antenna subset selection with space-time coding. IEEE Trans. Signal Process. 50, 2580-2588 (2002)

30. A Goldsmith, Wireless Communications. (Cambridge University Press, Cambridge, 2005)

31. MD Renzo, H Haas, A general framework for performance analysis of space shift keying (SSK) modulation for MISO correlated Nakagami-m fading channels. IEEE Trans. Commun. 58, 2590-2603 (2010)

32. J Proakis, Digital Communications, 4th edn. (McGraw-Hill, New York, 2000)

33. JA da Silva Ney, MLR de Campos, Spectrally efficient UWB pulse shaping with application in orthogonal PSM. IEEE Trans. Commun. 55, 313-322 (2007)

34. J Wang, S Jia, J Song, Generalised spatial modulation system with multiple active transmit antennas and low complexity detection scheme. IEEE Trans. Wireless Commun. 11, 1605-1615 (2012)

35. MK Simon, MS Alouini, Digital Communication over Fading Channels, 1st edn. (Wiley, Hoboken, 2000)

doi:10.1186/1687-1499-2013-43

Cite this article as: Ntontin et al.: Adaptive generalized space shift keying. EURASIP Journal on Wireless Communications and Networking 2013 2013:43.

\section{Submit your manuscript to a SpringerOpen ${ }^{\circ}$ journal and benefit from:}

- Convenient online submission

- Rigorous peer review

- Immediate publication on acceptance

- Open access: articles freely available online

- High visibility within the field

- Retaining the copyright to your article

Submit your next manuscript at $\boldsymbol{\wedge}$ springeropen.com 